\title{
Inhalt / Sommaire
}

Elisabeth Kloë und Hildburg Kindt, Zur Entstehung und Entwicklung des kindlichen Hysteriebegriffes

Erna Lesky, Der angeklagte Gall

Heinrich Stamm, 75 Jahre Société de Gynécologie et d'Obstétrique de la Suisse Romande und 70 Jahre Gynäkologische Gesellschaft der deutschen Schweiz

Jean Jacques Dreifuss, W. L. Gaines, précurseur du concept de réflexe neuroendocrine

Peter Speiser, Vogts Lehrbuch und Atlas der Spaltlampenmikroskopie des lebenden Auges

Ulrich Hoyer, Wellenmechanik und Boltzmannsche Statistik

Karl Heinrich Wiederkehr, Über die Entdeckung der Röntgenstrahlinterferenzen durch Laue und die Bestätigung der Kristallgittertheorie

\section{Buchbesprechungen}

Juan José Barcia Goyanes, Onomatologia Anatomica Nova (Erwin H. Ackerknecht)

P.Huard und M.J.Imbault-Huard, André Vésale: Iconographie anatomique (Erwin H. Ackerknecht)

Jakob Büchi, Die Arzneiverordnung und der Sanitätsdienst der Schweizer Truppen vom 15.-18. Jahrhundert (Erwin H. Ackerknecht)

Jacques Léonard, La médecine entre les pouvoirs et les savoirs (Erwin H. Ackerknecht)

Claude Bernard, Notes pour le Rapport sur les Progrès de la Physiologie

(Erwin H. Ackerknecht)

Georg Meissners Briefe an Jacob Henle 1855-1878 (Erwin H. Ackerknecht)

Wilfried Schungel, Alexander Tille (1860-1912) (Erwin H. Ackerknecht)

Käte Frankenthal, Der dreifache Fluch: Jüdin, Intellektuelle, Sozialistin

(Erwin H. Ackerknecht)

Kathleen M. Pearle, Preventive medicine: The Refugee Physician and the New York Medical Community 1933-1945 (Erwin H. Ackerknecht)

Achim Thom und Karl-Heinz Karbe, Henry Ernest Sigerist (1891-1957). Begründer einer modernen Sozialgeschichte der Medizin (Erwin H. Ackerknecht)

Heinrich Rodegra et al., Der Verband der niedergelassenen Ärzte Deutschlands (NAV) e.V.

Friedrich Rintelen, Geschichte der Medizinischen Fakultät in Basel 1900-1945 (Carl Haffter)

Geneviève Heller, Propre en ordre. Habitation et vie domestique 1850-1930:

l'exemple vaudoise (Carl Haffter)

Maria Elisabeth Wasserfuhr, Der Zahnarzt in der niederländischen Malerei des 17. Jahrhunderts (Peter Bigler) 